\title{
Micro-CT Imaging of RGD-Conjugated Gold Nanorods Targeting Tumor In Vivo
}

\author{
Xiaochao Qu, Xiaoxiao Li, Jingning Liang, Yanran Wang, Muhan Liu, and Jimin Liang \\ School of Life Science and Technology, Xidian University, Xian, Shaanxi 710071, China \\ Correspondence should be addressed to Xiaochao Qu; xiaochaoqu@gmail.com and Jimin Liang; jiminliang@gmail.com
}

Received 18 April 2016; Revised 21 June 2016; Accepted 22 June 2016

Academic Editor: Shuai Zhang

Copyright ( 2016 Xiaochao Qu et al. This is an open access article distributed under the Creative Commons Attribution License, which permits unrestricted use, distribution, and reproduction in any medium, provided the original work is properly cited.

\begin{abstract}
Gold nanomaterials as computed tomography (CT) contrast agents at lower X-ray dosage to get a higher contrast have advantages of longer imaging time and lower toxic side effects compared to current contrast agents. As a receptor for Cyclo (Arg-Gly-Asp-D-PheLys) (RGD) peptide, integrin $\alpha_{v} \beta_{3}$ is overexpressed on some tumor cells and tumor neovasculature. In this paper, we conjugated the RGD peptide on the surface of gold nanorods (AuNRs), designated as RGD-AuNRs, a promising candidate in applications such as tumor targeting and imaging capability for micro-CT imaging. Integrin $\alpha_{v} \beta_{3}$-positive U87 cells and integrin $\alpha_{v} \beta_{3}$-negative HT-29 cells were chosen to establish animal models relatedly and then texted the tumor targeting ability and imaging capability of RGDAuNRs in vitro and in vivo. The MTT assay and stability measurement showed that RGD-conjugation eliminated their cytotoxicity and improved their biocompatibility and stability. Dark-field imaging of U87 cells and HT-29 cells testified the binding affinities and uptake abilities of RGD-AuNRs, and the results showed that RGD-AuNRs were more specifical to U87 cells. The enhanced microCT imaging contrast of intramuscular and subcutaneous injection illustrated the feasibility of RGD-AuNRs to be contrast agents. Furthermore, the micro-CT imaging of targeting U87 and HT-29 tumor models verified the targeting abilities of RGD-AuNRs.
\end{abstract}

\section{Introduction}

Medical imaging plays an important role in overall cancer diagnostics and planning and analysis of the therapeutic effect. Some structural imaging modalities, such as computed tomography (CT), magnetic resonance imaging (MRI), and ultrasound imaging, are used to study the different biological processes of tumor and provide some basic information regarding tumor location, size, and shape. However, these imaging modalities rarely detect tumors smaller than $0.5 \mathrm{~cm}$, which limits their application in early clinical diagnosis [1]. In recent years, many different types of targeted nanomaterials, such as gold nanostructures [2-5], superparamagnetic nanoparticles [6-8], and ultrasound microbubbles $[9,10]$, have been developed for structural imaging modalities and help them acquire much higher resolution anatomic images.

Among all the imaging techniques, X-ray computed tomography (CT) is one of the most effective methods for the diagnosis and treatment of diseases in hospitals today for its availability, high efficiency, and low cost $[11,12]$. There is no doubt that $\mathrm{CT}$ is among the leading technologies in cancer management. CT is able to provide supplementary anatomical information to assist the diagnosis and treatment of the tumors [2]. Due to the fact that CT relies on the X-ray attenuation by the tissues, electron-dense parts could get better images. Compared to soft tissues, hard tissues have a higher $\mathrm{X}$-ray attenuation. However, the contrast between soft tissues is not obvious due to their similar CT values in the range from 0 through 50 Hounsfield Units (HU) [13]. In terms of the limitations of current contrast agents, CT imaging results are not satisfied. Hence, a major challenge in CT imaging of biological tissues is to achieve a high concentration of contrast agents gathered at the lesion site [14, 15]. Iodine containing molecules as contrast agents are commonly used in conventional CT imaging [14]. Such contrast agents have advantages in absorbing X-ray, but they do not possess the function of specific targeting due to the fact that they cannot conjugate to most biological components or identify cancer markers [3]. It is impossible for iodine containing molecules to accumulate at focus of infection at a concentration high enough to be 
detected by current CT scanners. High osmotic pressure and viscosity may cause side effects and renal toxicity; kidney failure has also been reported [16]. Moreover, due to the rapid renal clearance, they allow very short imaging times [17, 18]. Compared to traditional clinical CT equipment of high efficiency for conventional diagnosis in terms of their short response time (e.g., five seconds to capture effective information), micro-CT systems are often quite insensitive (e.g., ten minutes to scan a mouse) [16]. Therefore, X-ray contrast agents also need further development for better use.

It is imperative to develop contrast agents to overcome the shortcomings of current CT imaging. It is well known that CT contrast agents with high atomic number materials could produce better contrast effect than iodine-based contrast agents and have the ability to lower the radiation exposure to patients [19]. It has been reported that compared to iodine, gold has a higher attenuation coefficient and 2.7 times greater contrast effect than iodine at the same X-ray dose [20]. In addition to the advantages above, the applications of gold nanoparticles for cancer diagnosis and CT imaging are superior with the properties of easy modification in the surface to conjugate with specific functional molecules for targeting tumor cells (e.g., antibody and peptides) and the satisfactory biocompatibility [16]. In recent years, nanomaterials have obtained more attention due to its special optical properties and shape. With the change of the shape, gold nanorods (AuNRs) perform tunable optical properties which could be applied on biomedical targeting, photothermal therapy, and in vivo imaging $[21,22]$. More recently, AuNRs as a potential $\mathrm{X}$-ray contrast agent have been used in computed tomography (CT) imaging [23]. Although the CT contrast effect is not affected substantially by the shape of nanoparticles, gold nanorods have the advantage of being functionally modified. More importantly, the gold nanorods exhibit a better performance in evading clearance by phagocytes to get a longer circulation time than spherical nanoparticles [13]. Currently, iodine-based CT contrast agents imaging have a very short half-life time ( $<10 \mathrm{~min})$, and there is no specific targeting of the tumor. In the literatures, it has been reported that PEGylated gold nanorods can lead to a prolonged half-life time of $19 \mathrm{~h} \mathrm{[24].} \mathrm{On} \mathrm{the} \mathrm{other} \mathrm{hand,} \mathrm{to} \mathrm{increase} \mathrm{targeting} \mathrm{ability,}$ AuNRs can be functionalized by chemical modification such as DNA, peptides, and antibodies [2, 21, 25]. It is well known that integrin $\alpha_{v} \beta_{3}$ plays a decisive role in tumor growth, invasion, and metastasis. Integrin $\alpha_{v} \beta_{3}$ has a negligible expression on epithelial cells and mature endothelial cells, but it is highly upregulated on the activated endothelial cells of tumor neovasculature and some tumor cells such as human glioblastoma cell line (U87) $[26,27]$. It is noteworthy that peptides (RGD) could target integrin $\alpha_{v} \beta_{3}$, which can specifically track several kinds of tumors whereas antibody can only specifically recognize one certain kind of tumor.

Over the past decade, a series of (Arg-Gly-Asp-D-PheLys) (RGD) peptides based probes has been managed in the use of multimodal molecular imaging of integrin $\alpha_{v} \beta_{3}$ expression, and the RGD sequence probes with radionuclide have been used in clinical study $[27,28]$. Different from the extensive application of radiolabeled RGD peptide for tumor imaging, the RGD-based nanoprobes with targeting ability are usually rare [28].

In this paper, we obtained RGD-AuNRs nanoprobes with an ability of targeting tumor cells by modification with a bifunctional PEG and conjugation with RGD peptide. By cell viability assay and dark-field imaging, we verified the biocompatibility of RGD-AuNRs which facilitated the subsequent in vivo experiments. First, the comparison of micro-CT imaging between AuNRs and iohexol showed the advantage of AuNRs in terms of CT contrast effect. Second, to verify the feasibility of RGD-AuNRs for CT imaging in vivo, in vivo micro-CT imaging of intramuscular and subcutaneous injection of RGD-AuNRs was managed. Last, RGD-AuNRs were tail vein injected into U87 tumor model (with integrin $\alpha_{v} \beta_{3}$ positive tumor cells) and HT-29 tumor model (with integrin $\alpha_{v} \beta_{3}$-negative tumor cells). Then, micro-CT imaging of two tumor models were performed to detect the targeting ability of RGD-AuNRs and the imaging effect of the tumor site.

\section{Materials and Methods}

2.1. Materials and Reagents. Hydrogen tetrachloroaurate (III) trihydrate $\left(\mathrm{HAuCl}_{4} \cdot 3 \mathrm{H}_{2} \mathrm{O}\right)$, cetyl trimethyl ammonium bromide (CTAB), poly(ethylene glycol) 2-mercaptoethyl ether acetic acid (HS-PEG-COOH), Thiazolyl Blue Tetrazolium Bromide (MTT), and Dimethyl Sulfoxide (DMSO) hybridmax were purchased from Sigma-Aldrich (St. Louis, USA). Sodium borohydride $\left(\mathrm{NaBH}_{4}\right)$ was purchased from Kermel Chemical Reagent Co. Ltd. (Tianjin, China). L-ascorbic acid (AA) was obtained from Tianli Chemical Reagent (Tianjin, China). Silver nitrate $\left(\mathrm{AgNO}_{3}\right)$ was obtained from Shanghai Chemical Reagent Co. Ltd. (Shanghai, China). 1-Ethyl-3-(3-dimethylaminopropyl)carbodiimide (EDC) and N-hydroxysulfosuccinimide (Sulfo-NHS) were purchased from Aladdin (Shanghai, China). Cyclo (Arg-Gly-Asp-DPhe-Lys) (RGD) was prepared by GL Biochem Co. Ltd. (Shanghai, China). All the above chemicals were used without any further purification. Deionized water (Millipore Milli-Q grade) with a resistivity of $18.2 \mathrm{M} \Omega \mathrm{cm}^{-1}$ was used in all the preparations.

2.2. Preparation of AuNRs. AuNRs was prepared based on the seed-mediated growth method of Nikoobakht and ElSayed [29]. Seed solution was needed to prepare AuNRs. $5 \mathrm{~mL}$ of $\mathrm{HAuCl}_{4}(0.5 \mathrm{mM})$ was added into $5 \mathrm{~mL}$ of CTAB solution $(0.2 \mathrm{M})$. Then, $0.6 \mathrm{~mL}$ of $\mathrm{NaBH}_{4}(0.01 \mathrm{M})$ at $0^{\circ} \mathrm{C}$ was added. The seed solution was vigorously stirred for $2 \mathrm{~min}$ and kept at $25^{\circ} \mathrm{C}$. Then, we prepared growth solution. $0.25 \mathrm{~mL}$ of $\mathrm{AgNO}_{3}$ solution $(0.004 \mathrm{M})$ was mixed with $5 \mathrm{~mL}$ of CTAB solution $(0.2 \mathrm{M})$ at $25^{\circ} \mathrm{C} .5 \mathrm{~mL}$ of $\mathrm{HAuCl}_{4}(1 \mathrm{mM})$ was added into the mixture, and then $70 \mu \mathrm{L}$ of ascorbic acid $(0.0788 \mathrm{M})$ was added which changed the color of solution from yellow to colorless. Finally, $12 \mu \mathrm{L}$ of the seed solution was injected into the growth solution, then standing for $3 \mathrm{~h}$ at $27.5^{\circ} \mathrm{C}$. The product was kept at $4^{\circ} \mathrm{C}$ for further use.

2.3. PEGylation and RGD Conjugation. In order to prepare RGD-conjugated AuNRs, CTAB on the surface of the gold nanorods needed to be replaced with bifunctional PEG 


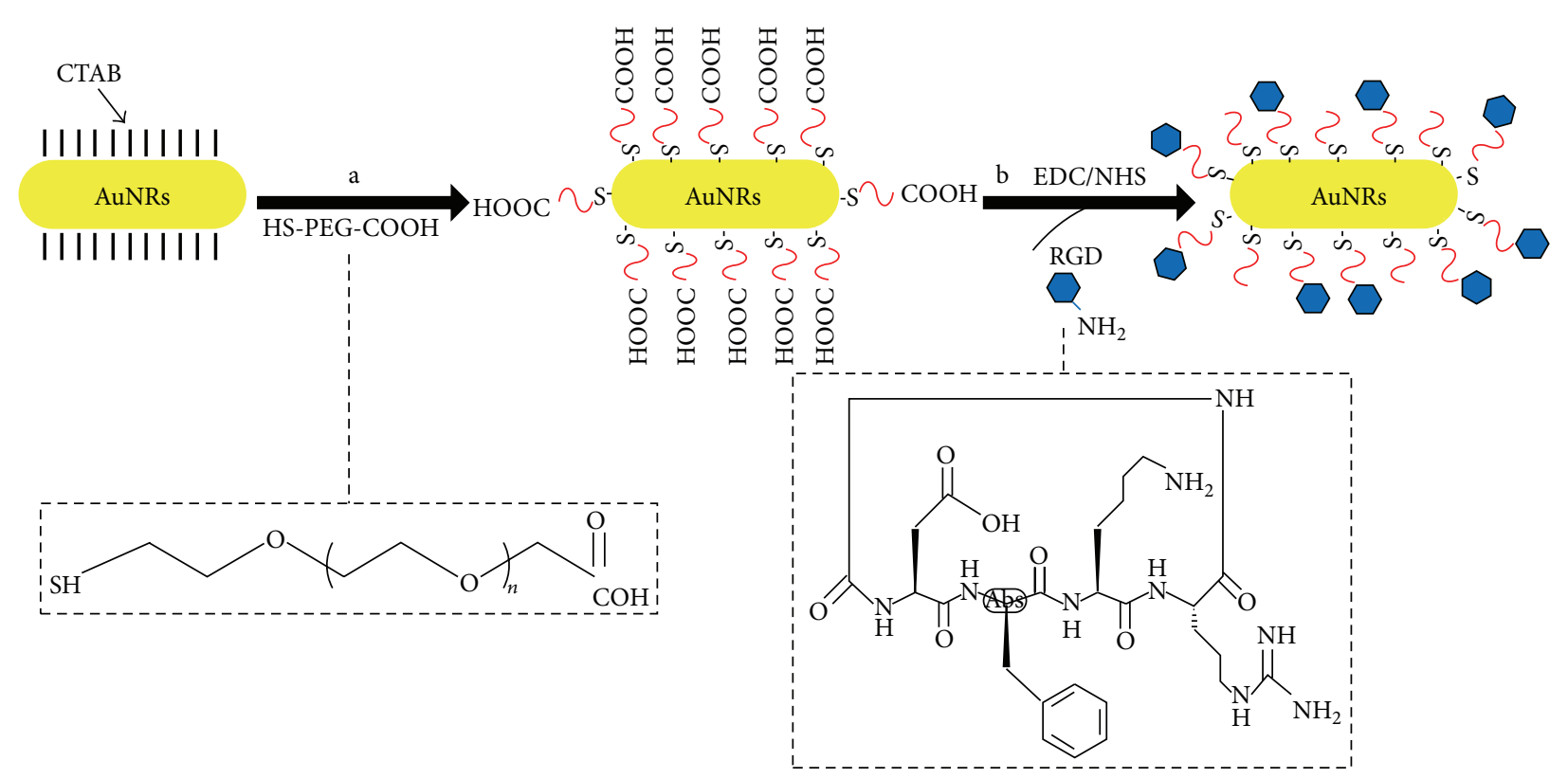

FIGURE 1: Schematic illustration of the synthesis of RGD-conjugated PEGylated AuNRs. a: CTAB was substituted for a bifunctional PEG. b: RGD was conjugated to PEG-AuNRs.

[30]. Figure 1 shows the synthetic pathway for preparing RGD-conjugated PEGylated AuNRs. (i) $30 \mathrm{mg}$ of HS-PEG$\mathrm{COOH}(\mathrm{MW}=5 \mathrm{k})$ was added into $2 \mathrm{~mL}$ of gold nanorods solution $\left(\mathrm{C}_{\mathrm{Au}}=300 \mu \mathrm{g} / \mathrm{mL}\right)$ and then stirred for $24 \mathrm{~h}$ at $25^{\circ} \mathrm{C}$. To remove unreacted PEG, the mixture was centrifuged at $11,000 \mathrm{rpm}$ for $10 \mathrm{~min}$ and then was resuspended in $2 \mathrm{~mL}$ of phosphate buffered saline (PBS) $(\mathrm{pH}=7.4)$. (ii) $16.91 \mathrm{mg}$ of EDC (1-ethyl-3-(3-dimethylaminopropyl)carbodiimide) and $10.15 \mathrm{mg}$ of Sulfo-NHS (N-hydroxysulfosuccinimide) were added into the mixture for $30 \mathrm{~min}$ to activate the carboxyl groups on the surface of AuNRs and stabilize the activated groups of carboxyl. The mixture was centrifuged at $11,000 \mathrm{rpm}$ for $10 \mathrm{~min}$ to remove the excess EDC and Sulfo-NHS and resuspended in $2 \mathrm{~mL}$ of PBS. (iii) The final step was to conjugate RGD (MW $=5 \mathrm{k}$ ). $500 \mu \mathrm{L}$ of RGD $(1.656 \mu \mathrm{mol} / \mathrm{mL})$ was added to $2 \mathrm{~mL}$ of PEGylation-AuNRs (PEG-AuNRs) solution and reacted at $4^{\circ} \mathrm{C}$ for $6 \mathrm{~h}$. After the reaction, excess RGD was removed by centrifugation at $11,000 \mathrm{rpm}$ for $10 \mathrm{~min}$ and resuspended in $1 \mathrm{~mL}$ of PBS.

2.4. Characterization of Gold Nanomaterials. To characterize the gold nanomaterials, zeta potentials were measured with a Zetasizer Nano ZS in water (Malvern Zen 3690, United Kingdom) and UV-Vis spectrums were measured at $25^{\circ} \mathrm{C}$ by the SHMADZU UV-2450 UV-visible spectrophotometer with $1 \mathrm{~cm}$ quartz cell. Transmission electron microscope measurements were performed by H-600 (Hitachi, Japan) operated at $75 \mathrm{kV}$ accelerating voltage.

2.5. Cell Culture. Human glioblastoma cell line (U87) (integrin $\alpha_{v} \beta_{3}$-positive tumor cells) and human colon malignant tumor cell line (HT-29) (integrin $\alpha_{v} \beta_{3}$-negative tumor cells) were cultured in 90\% Dulbecco's Modified Eagle's Medium (DMEM) of high glucose culture medium (Hyclone,
Thermo Scientific, US) with the addition of $10 \%$ fetal bovine serum (Hyclone, Thermo Scientific, US) and $1 \%$ antibiotics (Hyclone, Thermo Scientific, US). Temperature and carbon dioxide content were maintained at $37^{\circ} \mathrm{C}, 5 \%$.

2.6. MTT Assay. To certify the biocompatibility of gold nanomaterials, cell viability assays were managed. The U87 and HT-29 cells were inoculated into 96-well plates $(5,000$ cells per well), respectively, and incubated for $24 \mathrm{~h}$. The sterile AuNRs and RGD-AuNRs were added to 96-well plates (200 $\mu \mathrm{L}$ per well) at different concentrations $(100,10,1,0.1$, 0.01 , and $0.001 \mathrm{nmol} / \mathrm{mL}, \mathrm{Au})$. Then, The U87 and HT-29 cells were incubated for another $72 \mathrm{~h}$. After incubation, the cells were exhaustively washed with sterile PBS to remove residual gold nanomaterials. $20 \mu \mathrm{L}$ MTT $(5 \mathrm{mg} / \mathrm{mL})$ was added to each well subsequently and incubated for $4 \mathrm{~h} .150 \mu \mathrm{L}$ DMSO was added to each well to dissolve the formazan crystals and then the medium was shocked for $15 \mathrm{~min}$. Reading was taken in Spectramax 190 (Molecular Devices, China) at $490 \mathrm{~nm}$.

2.7. Dark-Field Imaging. To evaluate integrin $\alpha_{v} \beta_{3}$ binding affinities and uptake abilities of gold nanomaterials with tumor cells, the U87 and HT-29 cells were chosen and incubated for 3 days. After trypsinized, the cells were centrifuged at $800 \mathrm{rpm}$ for $5 \mathrm{~min}$. The supernatant was discarded, and the solid was suspended in $2 \mathrm{~mL}$ culture medium which was then distributed into two sterile tubes homogeneously. $200 \mu \mathrm{L}$ of PEG-AuNRs and RGD-AuNRs $\left(C_{\mathrm{Au}}=600 \mu \mathrm{g} / \mathrm{mL}\right)$ were added to U87 cells, respectively, with HT-29 cells being the same case. Then, U87 and HT-29 cells were incubated in cell incubator for $30 \mathrm{~min}$ at $37^{\circ} \mathrm{C}$. The cells were rinsed with PBS twice and then resuspended in $200 \mu \mathrm{L}$ PBS to remove unbound nanomaterials. Glass slides were prepared and 
fastened with clean-mounts. An Olympus IX70 microscope at 40x magnification was used to perform dark-field imaging.

\subsection{Micro-CT Imaging of Gold Nanomaterials}

2.8.1. Micro-CT Imaging of AuNRs. Different concentrations of AuNRs $\left(C_{A u}=1,5\right.$ and $\left.10 \mathrm{mg} / \mathrm{mL}\right)$, iohexol $(10 \mathrm{mg} / \mathrm{mL})$, and deionized water were prepared in $200 \mu \mathrm{L}$ tubes. Tubes were divided into three groups, including experiment group (AuNRs), control group (iohexol), and blank (deionized water), and then scanned by a micro-CT imaging system which consisted of a microfocus X-ray source (Apogee, Oxford Instruments) and an X-ray flat panel detector (C7921CA-02, Hamamatsu, Japan), with the following operating parameters of $50 \mathrm{kV}, 1 \mathrm{~mA}$ with a $512 \times 512$ matrix size, and 360 views.

2.8.2. Feasibility Verification of In Vivo Micro-CT Imaging of RGD-AuNRs. Female mice weighing about $27 \mathrm{~g}$ were purchased from the Department of Experimental Animal of Xian Jiaotong University. The animals were treated in accordance with institutional animal use and care regulations. After the mouse was anesthetized (with $0.1 \mathrm{~mL} 10 \%$ chloral hydrate and $1 \%$ atropine), $100 \mu \mathrm{L}$ of RGD-AuNRs in PBS was intramuscularly injected into the right hind leg area $\left(\mathrm{C}_{\mathrm{Au}}=10 \mathrm{mg} / \mathrm{mL}\right)$ and subcutaneously injected into the back of mouse $\left(\mathrm{C}_{\mathrm{Au}}=5 \mathrm{mg} / \mathrm{mL}\right)$, respectively. The mouse was fixed on a homemade holder using the above micro-CT imaging system with parameter setting unchanged. As a control contrast, the mouse was scanned before RGD-AuNRs injection. And 10 days after injection, no proof of toxicity was observed.

2.8.3. In Vivo Micro-CT Imaging of RGD-AuNRs Targeting Tumor Mouse. Two nude mice were injected with cancer cells $\left(2 \times 10^{6}\right)$ into the right arm and divided into two groups including positive group (U87 cells) and negative group (HT29 cells), until the tumor reached a diameter of over $0.5 \mathrm{~cm}$. $100 \mu \mathrm{L}$ PBS solution of RGD-AuNRs $\left(\mathrm{C}_{\mathrm{Au}}=3 \mathrm{mg} / \mathrm{mL}\right)$ was tail vein injected into the two nude mice, respectively. The first scanning was carried out before $(0 \mathrm{~h})$ tail vein injection by NanoScan PET/CT (Mediso, Medical Imaging Systems, USA) with operating parameters of $55 \mathrm{kV}$ and $146 \mu \mathrm{A}$ to determine the CT datum line. CT scanning was performed after tail vein injection of RGD-AuNRs at time points of 1 and $3 \mathrm{~h}$. All CT scans were performed under gases of isoflurane anesthesia. The contrast effect was quantitatively determined by the differential contrast in CT values (in $\mathrm{HU}$ ).

\section{Results}

3.1. Characterization of the Nanoparticles. The characteristics of the gold nanomaterials are shown in Figure 2. And the UV-visible absorption spectrums of gold nanomaterials are presented in Figure 2(a). Compared to the spectrum of AuNRs, there was a minimal redshift in the longitudinal peak when conjugated with RGD, similar to PEGylation-AuNRs (PEG-AuNRs). The absorption spectrums demonstrated that the AuNRs, even after modification with HS-PEG-COOH and RGD, were able to maintain their morphology and optical

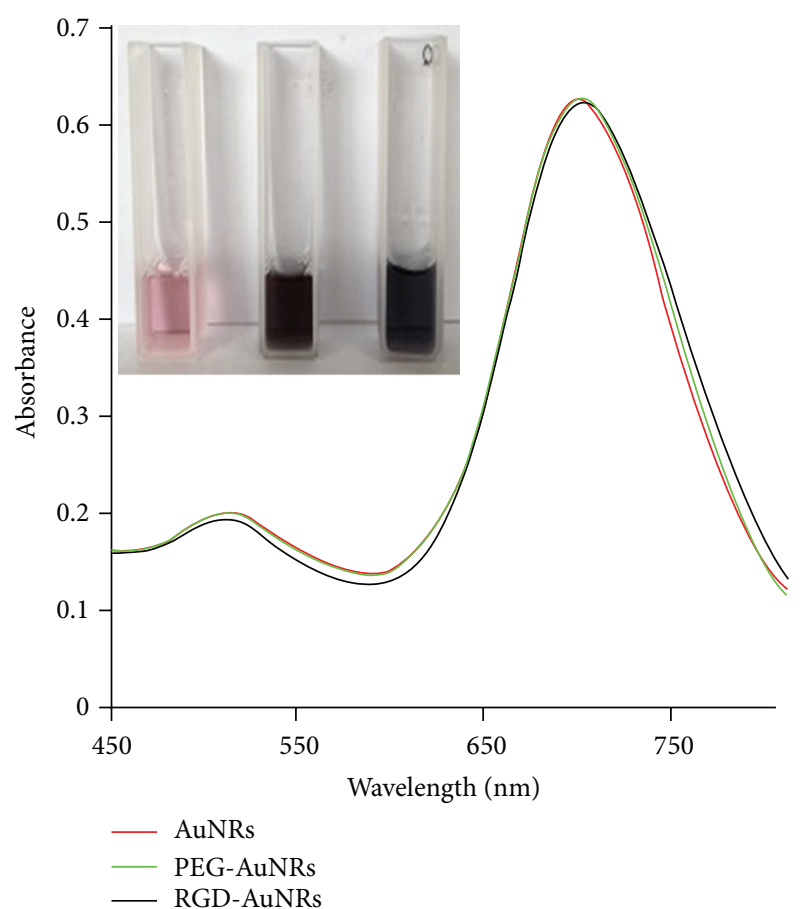

(a)

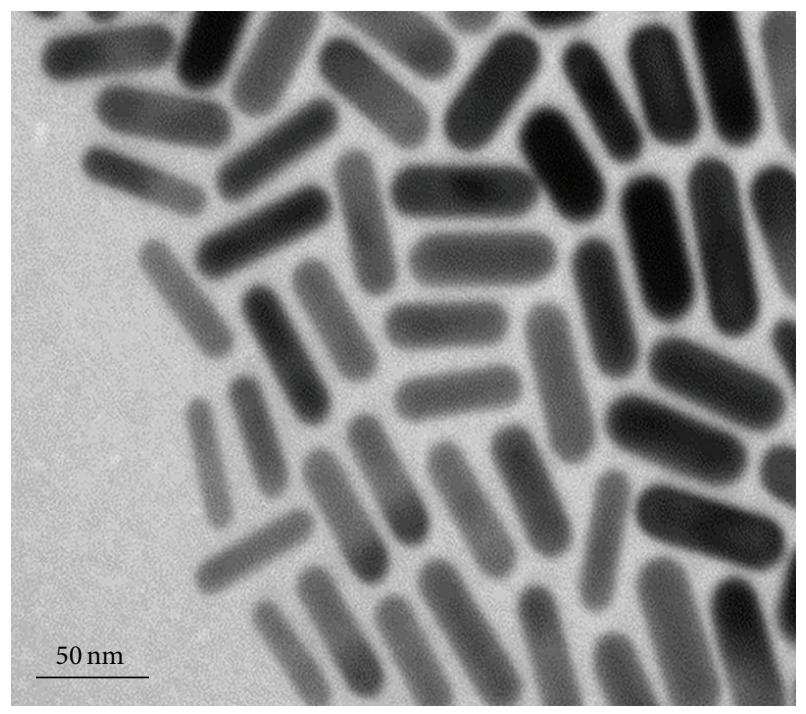

(b)

FIgUre 2: (a) UV-Vis spectrums of the AuNRs, PEG-AuNRs, and RGD-AuNRs (inset, the color changes from left to right are AuNRs, PEG-AuNRs, and RGD-AuNRs). (b) TEM image of AuNRs with plasmon band energies at $700 \mathrm{~nm}$, and the scale bar is $50 \mathrm{~nm}$.

properties in the NIR region. The structure and size of AuNRs were evaluated by TEM, as shown in Figure 2(b). The AuNRs had a good rod shape with a smooth surface, with aspect ratio (AR) of 2.5. At each step, surface potentials of AuNRs which changed with surface ligands changing are shown in Table 1. As declared in Figure 1, the AuNRs were first stabilized by HS-PEG-COOH and then by RGD in a sequential manner. The surface of the synthesized AuNRs 
TABLE 1: Zeta potentials of gold nanomaterials.

\begin{tabular}{lcc}
\hline \multirow{2}{*}{ Gold nanomaterials } & \multicolumn{2}{c}{ Zeta potential } \\
& Mean $(\mathrm{mV})$ & Standard deviation \\
\hline AuNRs & 40 & \pm 5 \\
PEG-AuNRs & -25 & \pm 2 \\
RGD-AuNRs & -17 & \pm 2 \\
\hline
\end{tabular}

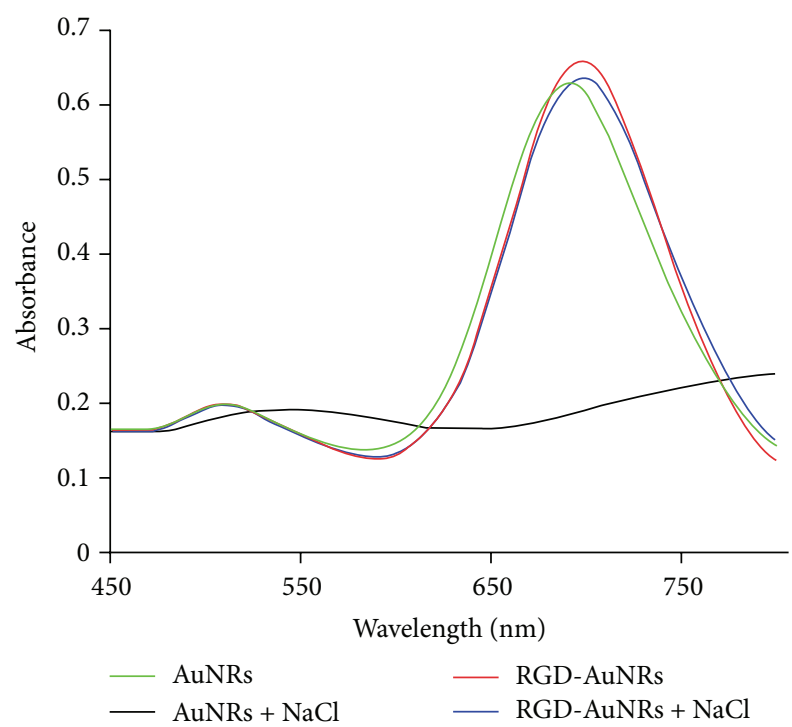

Figure 3: UV-Vis spectrums of AuNRs and RGD-AuNRs with different dispersants (deionized water and $10 \% \mathrm{NaCl}$ solution) at $4^{\circ} \mathrm{C}$.

was wrapped in $\mathrm{CTAB}$ with a positive surface charge of $40 \mathrm{mV}$. The HS-PEG-COOH substituting step decreased the surface potential to about $-25 \mathrm{mV}$, whereas RGD conjugation increased the zeta potential roughly to a degree of $-17 \mathrm{mV}$. The potential variation suggested that RGD was successfully conjugated with PEG-AuNRs.

3.2. Stability of RGD-AuNRs. The test of stability of RGDAuNRs was very important for their applications in vivo. Gormley et al. [31] investigated that the aggregation extent of RGD-AuNRs could be reported by the different absorption properties in the UV-Vis spectrum. So the stability of RGDAuNRs was analyzed via UV-Vis spectrometry (Figure 3). It could be seen that in the deionized water the spectrums of RGD-AuNRs and AuNRs did not show major changes. However, it was a different case when they were dispersed into $10 \% \mathrm{NaCl}$. RGD-AuNRs dispersed into $10 \% \mathrm{NaCl}$ had a similar spectrum as deionized water. But the spectrum of AuNRs dispersed into $10 \% \mathrm{NaCl}$ changed obviously, which suggested that AuNRs had aggregated and were unstable. The results indicated that RGD-conjugated AuNRs significantly improved the stability of the particles.

3.3. The Effects of RGD-AuNRs on Cytotoxicity In Vitro. After studying the RGD-AuNRs characteristics, we examined the extent of cytotoxicity to tumor cells. During the AuNRs preparation process, the surface stabilizer CTAB has a strong cytotoxicity, so it was necessary to analyze the cytotoxicity of RGD-AuNRs. The MTT assay results of U87 and HT29 cells, which are showed by open bars in Figure 4, both indicated that when the concentration of AuNRs (here, we actually referred to the concentration of $\mathrm{Au}$ ) was higher than $0.1 \mathrm{nM}$, there was a high cytotoxicity (lower than $80 \%$ viability). When the concentration was greater than $1 \mathrm{nM}$, the cell viability decreased apparently. On the other hand, due to the conjugation of RGD, no significant effects on the cell viability (higher than $80 \%$ viability) were seen even at a high concentration of $10 \mathrm{nM}$, as shown by closed bars in Figure 4. The data indicated that RGD conjugation significantly improved the biocompatibility of AuNRs for biomedical applications, especially when the concentration of particles required was high. RGD-AuNRs possessed a property of noncytotoxicity in the given concentration range and should be suitable for CT imaging applications in vivo.

3.4. The Ability of RGD-AuNRs Binding Tumor Cells. The ability of RGD-AuNRs to binding with tumor cells was evaluated by dark-field imaging, as shown in Figure 5. In terms of some tumor cells that had an expression of integrin $\alpha_{v} \beta_{3}$ to varying degrees, our tests used human glioblastoma line, U87 $\left(10^{4}\right.$ cells $/ \mathrm{mL}$, Figures $5(\mathrm{a}), 5(\mathrm{c})$, and $\left.5(\mathrm{e})\right)$, as a positive group and human colon cancer cell line, HT-29 $\left(10^{4}\right.$ cells $/ \mathrm{mL}$, Figures 5(b), 5(d), and 5(f)), as a negative group for comparison purpose. $150 \mu \mathrm{L}$ of PEG-AuNRs and RGD-AuNRs $\left(\mathrm{C}_{\mathrm{Au}}=\right.$ $0.4 \mathrm{nM}$ ) were added to each group, respectively. Experimental results showed that integrin $\alpha_{v} \beta_{3}$ had a high expression in the positive group. And peptides RGD on the surface of AuNRs had a strong ability to identify U87 tumor cells, as shown in Figure 5(c). Especially in the amplified image, after incubation with RGD-AuNRs, U87 cells displayed an apparently golden color around the cells, indicating aggregation of gold nanomaterials in the cells (Figure 5(e)). However, in the negative group, HT-29 cells with a low expression of integrin $\alpha_{v} \beta_{3}$ led to a poor binding ability of RGD, as shown in Figures 5(d) and 5(f). Meanwhile, PEG-AuNRs could not effectively recognize tumor cells, so the cells in the two experiments did not show obvious binding in dark-field imaging (Figures 5(a) and 5(b)). Finally, the binding ability of the RGD peptide was directly related to the expression ability of integrin $\alpha_{v} \beta_{3}$. With the higher expression of integrin $\alpha_{v} \beta_{3}$ on the tumor cells, it was easier for RGD-AuNRs to recognize tumor cells which provided us with excellent supporting for in vivo tumor targeting micro-CT imaging.

\subsection{Micro-CT Imaging of Gold Nanoparticles}

3.5.1. Micro-CT Imaging of AuNRs. AuNRs have a strong $\mathrm{X}$-ray attenuation, which are promising nanomaterials as contrast agents for CT imaging. In our study, the Hounsfield Units (HU) of AuNRs, iohexol, and deionized water were measured by micro-CT imaging system. Figure 6 shows the CT images and the attenuation values (in $\mathrm{HU}$ ) of each 


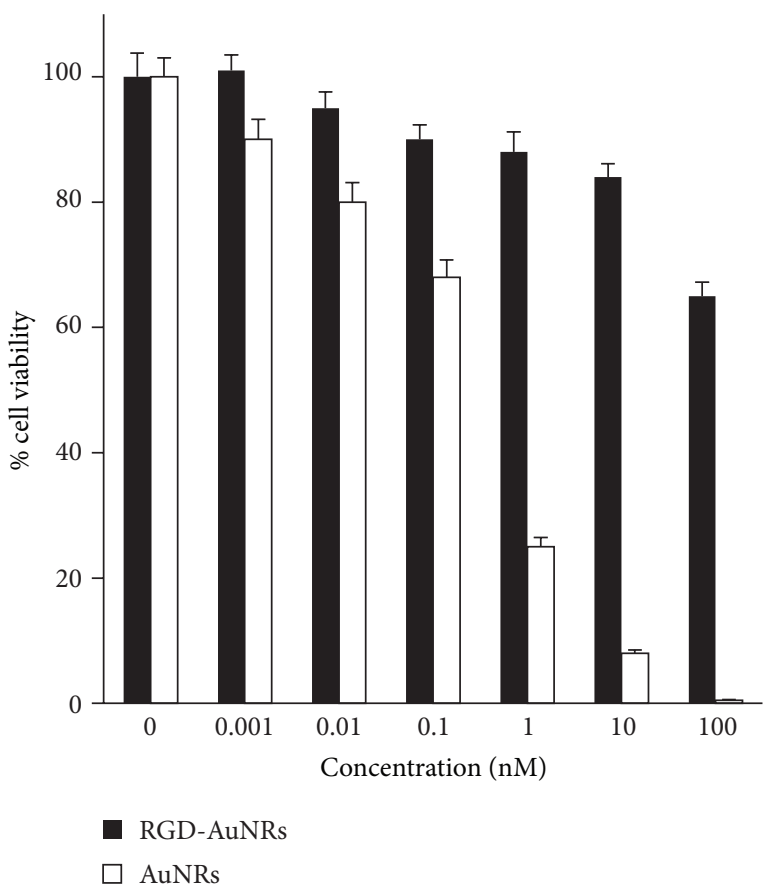

(a)

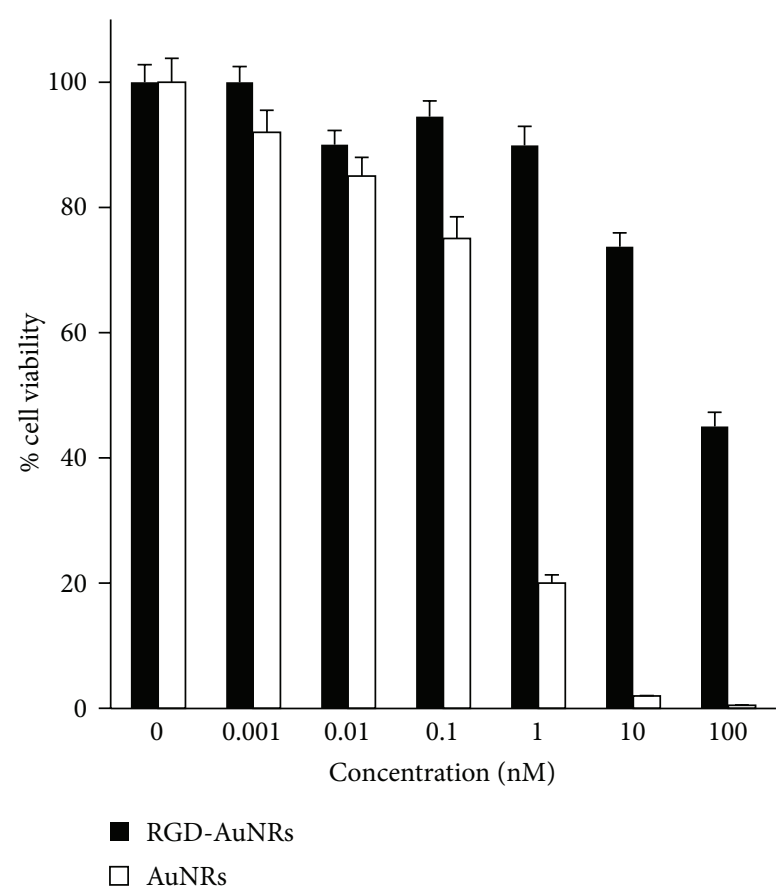

(b)

FIGURE 4: Viabilities of U87 cells (a) and HT-29 cells (b) after incubation with RGD-AuNRs (closed bars) and AuNRs (open bars). All data are averages for $n=3$. The error bars represent one standard deviation.

sample. The higher the concentration of AuNRs was, the brighter the images were, which indicated an increased $\mathrm{X}$ ray attenuation. HU quantitatively showed the proportional relationship between the concentration of AuNRs and CT signal intensity. When the concentration of iohexol and AuNRs kept the same level, a higher HU value of AuNRs was shown, which indicated that at the same X-ray dose, AuNRs would be more ideal as CT imaging contrast agents.

\subsubsection{Feasibility Verification of In Vivo Micro-CT Imaging of} $R G D$-AuNRs. To verify the feasibility of RGD-AuNRs for CT imaging in vivo, $100 \mu \mathrm{L}$ RGD-AuNRs in PBS was intramuscularly injected into the right hind leg area $\left(\mathrm{C}_{\mathrm{Au}}=10 \mathrm{mg} / \mathrm{mL}\right)$ and subcutaneously injected into the back of mouse $\left(\mathrm{C}_{\mathrm{Au}}=\right.$ $5 \mathrm{mg} / \mathrm{mL}$ ), respectively. And then the mouse was placed on a homemade holder after anesthesia and scanned by microCT imaging system. As shown in Figure 7, compared with other soft tissues, the back and leg area with injection had a contrast enhancement, which was attributed to the strong Xray attenuation of AuNRs. Before and after injection, the CT values of the injection sites were illustrated in Figure 8. The corresponding injection sites of the mouse showed a clearly distinguished CT signal intensity and a significant change in brightness which indicated a direct evidence of the imaging effect of RGD-AuNRs as contrast agents for CT imaging. And with a higher concentration of RGD-AuNRs, the brightness of the injection site was higher. CT values (in $\mathrm{HU}$ ) changed obviously from 11 to 100 (back) (Figures 8(a) and 8(c)) and 9 to 186 (right hind leg) (Figures 8(b) and 8(d)). Up to 10 days after injection, the mouse's behavior was normal which indicated that RGD-AuNRs were harmless to mouse.

\subsubsection{In Vivo Micro-CT Imaging of RGD-AuNRs Targeting}

Tumor Mouse. Human glioblastoma cells (U87) as a positive group and human colon cancer cells (HT-29) as a negative group were inoculated in nude mice. In our study, CT imaging was used to detect the ability of RGD-AuNRs to target tumor cells and tumor neovasculature during the whole blood circulation. Figure 9 displayed micro-CT images before and after ( 1 and $3 \mathrm{~h}$ ) intravenous injection of RGD-AuNRs in nude mice. $1 \mathrm{~h}$ after injection, tumor uptake of RGD-AuNRs was lower in HT-29 colon malignant tumor compared with U87 glioblastoma tumor, shown in Figures $9(\mathrm{c})$ and $9(\mathrm{~g})$. After injection of RGD-AuNRs $3 \mathrm{~h}$, there was a difference in brightness of images between positive group and negative group and the tumor site displayed an obvious contrast to be visualized compared with other soft tissues in positive group (Figures 9(d) and 9(h)). This result also showed U87 glioblastoma tumor had a higher expression of integrin $\alpha_{v} \beta_{3}$ than HT-29 colon malignant tumor. However, the contrast effects were still not as obvious as that in intramuscular and subcutaneous injection experiments (Figures 7 and 8). As shown in Figures 9(b)-9(d), with blood circulation, RGDAuNRs gradually targeted the tumor tissue during $0 \sim 3 \mathrm{~h}$, which was proved by the growing brightness of images. These results directly certified the practicality of U87 cells with high expression of integrin $\alpha_{v} \beta_{3}$ targeted by RGD-AuNRs with a high specificity. Meanwhile, AuNRs could be used as 


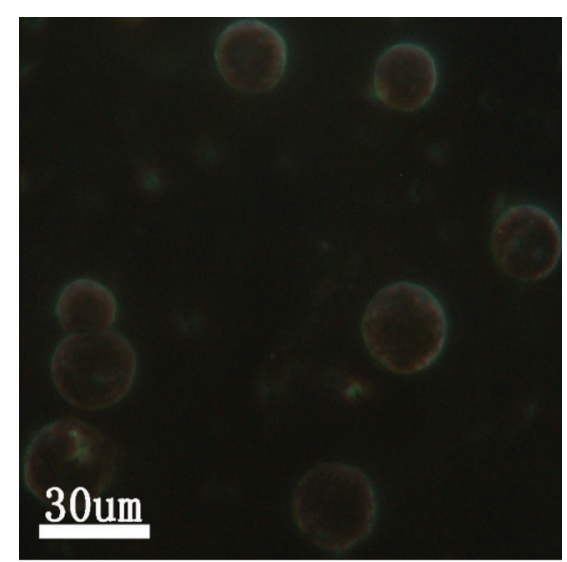

(a)

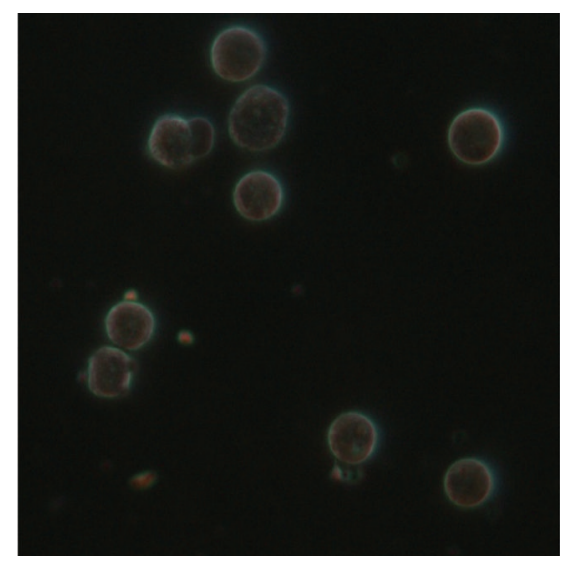

(d)

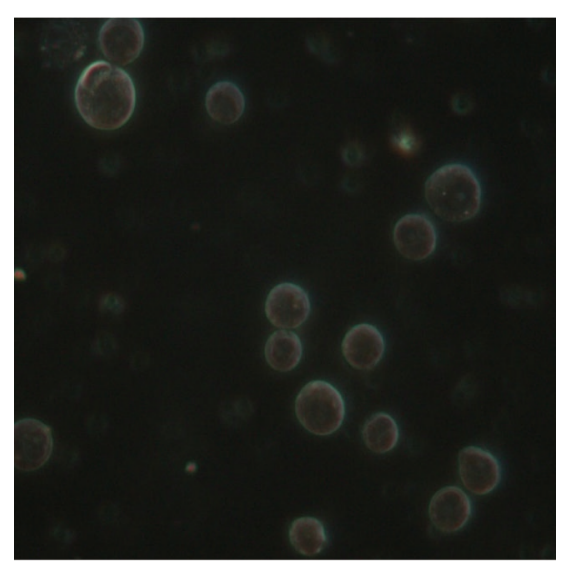

(b)

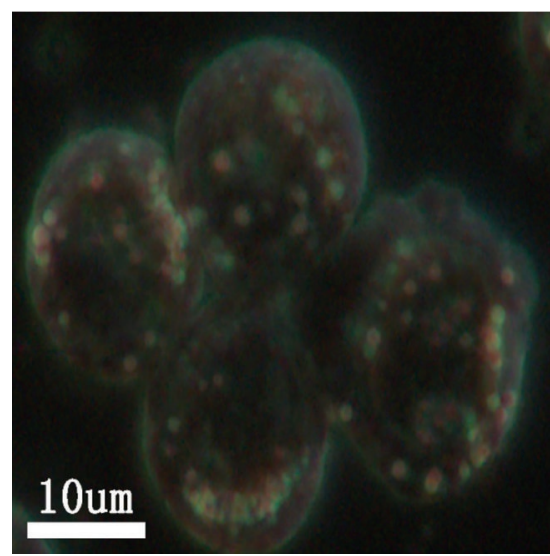

(e)

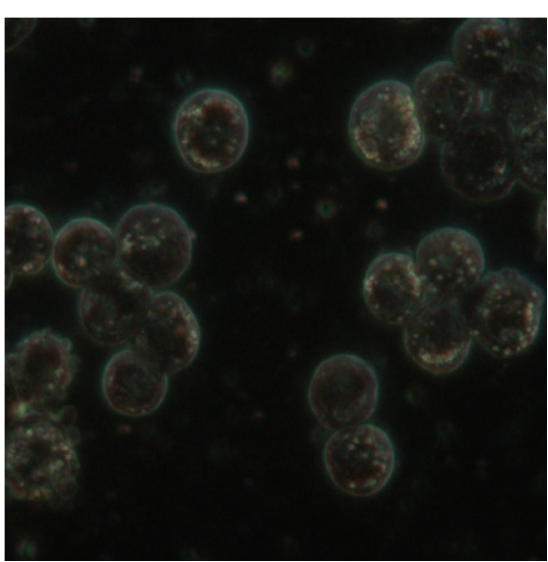

(c)

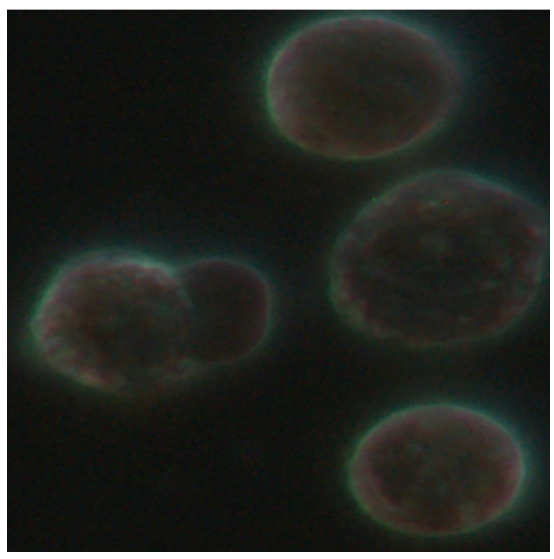

(f)

FIGURE 5: Dark-field imaging of U87 cells and HT-29 cells. (a) PEG-AuNRs with U87 cells. (b) PEG-AuNRs with HT-29 cells. (c) RGD-AuNRs with U87 cells. (d) RGD-AuNRs with HT-29 cells. (e) The corresponding amplified image of U87 cells in (c). (f) The corresponding amplified image of HT-29 cells in (d). Integrin $\alpha_{v} \beta_{3}$ expression in U87 cells is positive, so the brightness of the RGD-AuNRs targeting U87 cells in the dark-field imaging is highest. The scale bar of (a-d) is $30 \mu \mathrm{m}$ with (e-f) $10 \mu \mathrm{m}$.

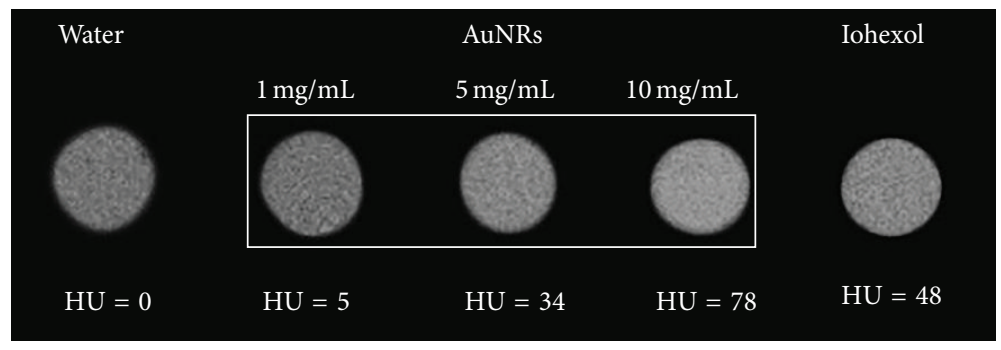

FIGURE 6: Tubes CT images of water, AuNRs, and iohexol. The concentration (mg/mL) of major elements (Au and I) in each tube is provided at the top of the image. The CT attenuation (in HU) in each tube is provided at the bottom of the image.

a potential contrast agent at the U87 tumor site to enhance $\mathrm{X}$-ray signal for CT imaging.

\section{Discussion}

X-ray computed tomography has advantages of high spatial resolution, wide range of applications, easy operation, low cost, and so forth. So far CT imaging is still the most basic and primary tumor pathological examination method, and even the preferred screening method for tumors on some parts of the bone tissues. Since the 1970s, iodine containing molecules as CT imaging contrast agents have been widely applied on CT imaging. But with the development of clinical medicine, iodine containing molecules as CT imaging contrast agents in imaging depth, imaging time, and specificity of imaging have been unable to satisfy existing imaging requirements. Since 


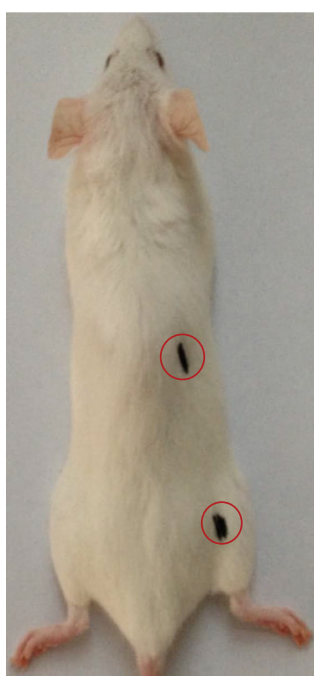

(a)

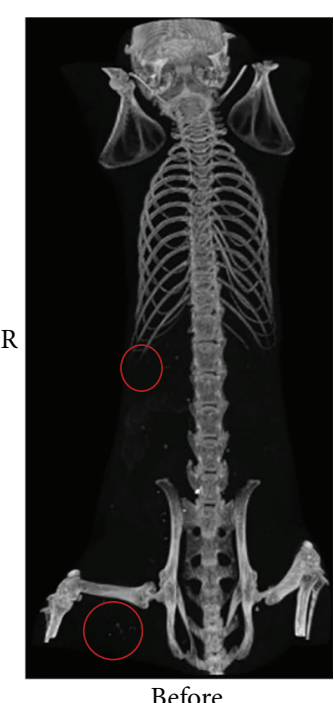

(b)

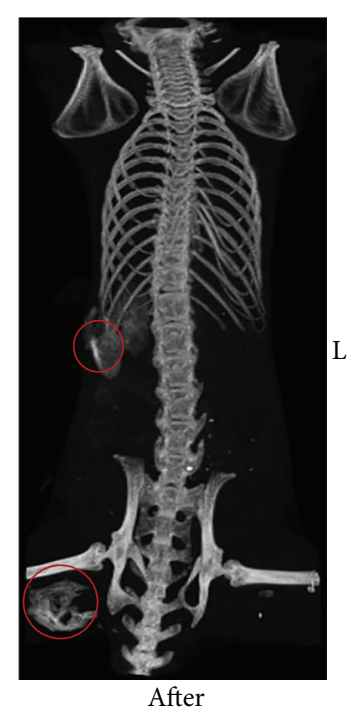

(c)

FIGURE 7: In vivo CT imaging of mouse before and after intramuscular and subcutaneous injection with different concentration of RGDAuNRs at different sites. (a) The photograph of mouse. (b) CT image before injection. (c) CT image after injection.

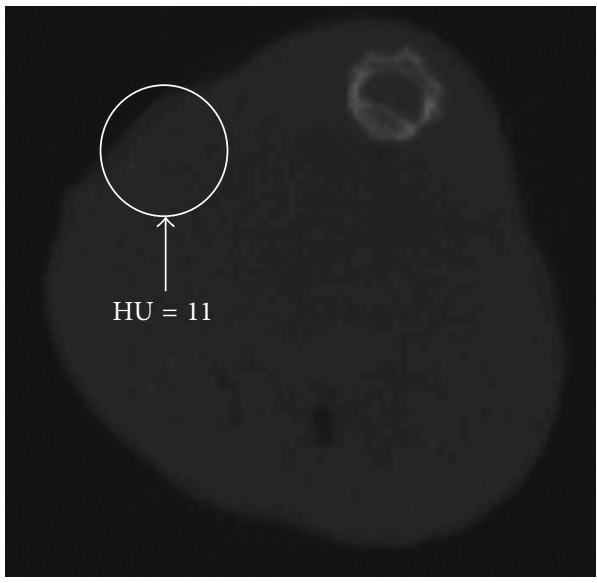

(a)

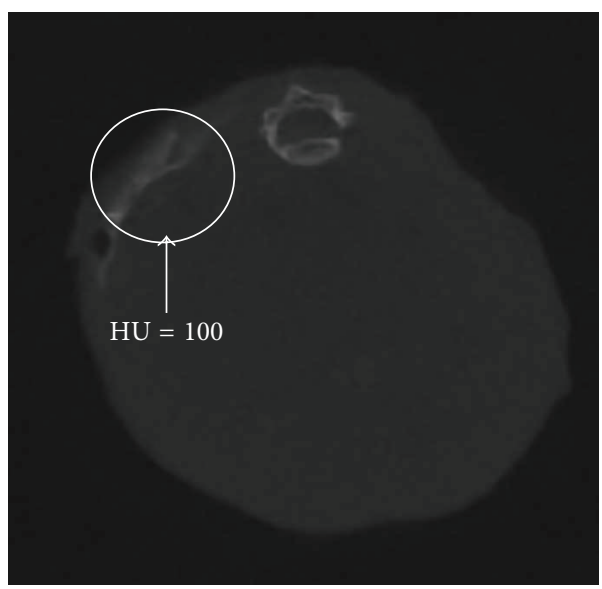

(c)

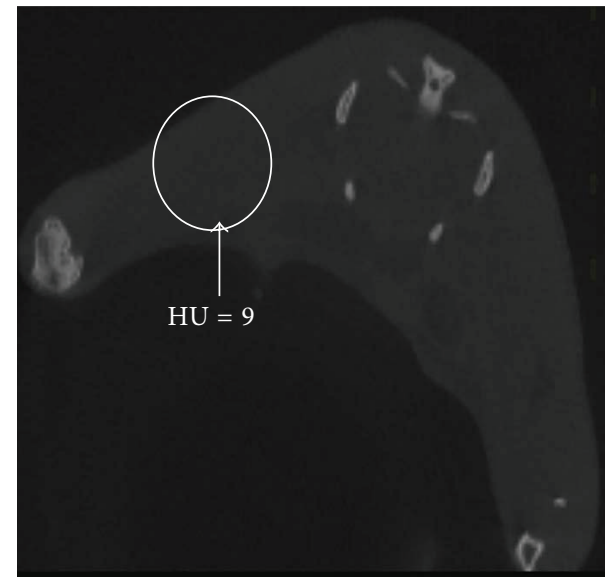

(b)

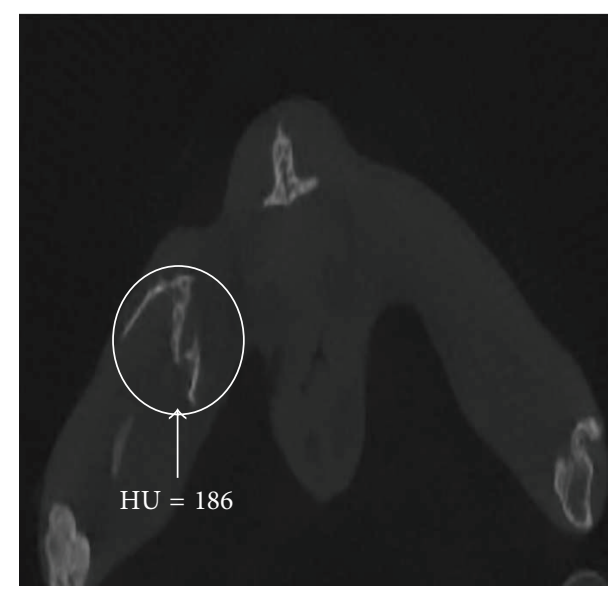

(d)

FIGURE 8: CT slices of mouse before and after injection with RGD-AuNRs. (a, c) The transverse slice images of the back area, the HU values of the injection site changed from 11 to 100. (b, d) The transverse slice images of the right hind leg, the HU values of the injection site changed from 9 to 186. 


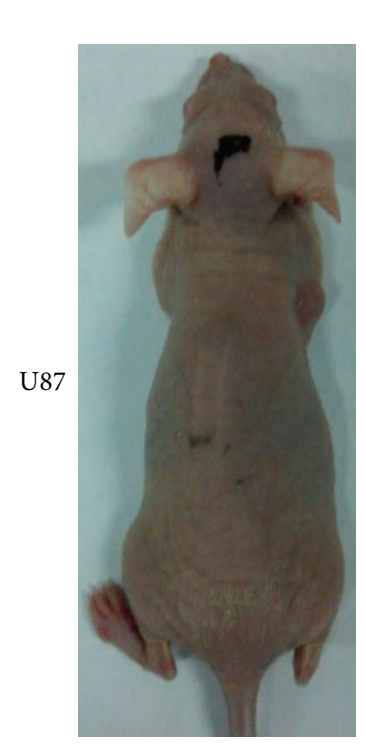

(a)

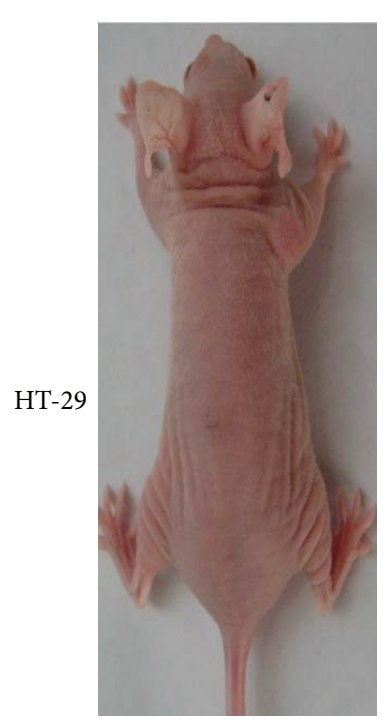

(e)

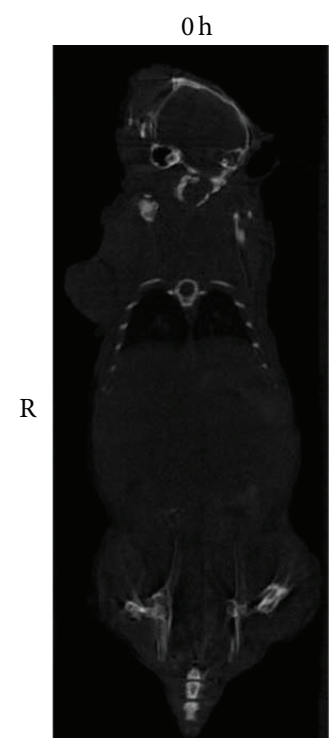

(b)

$0 \mathrm{~h}$

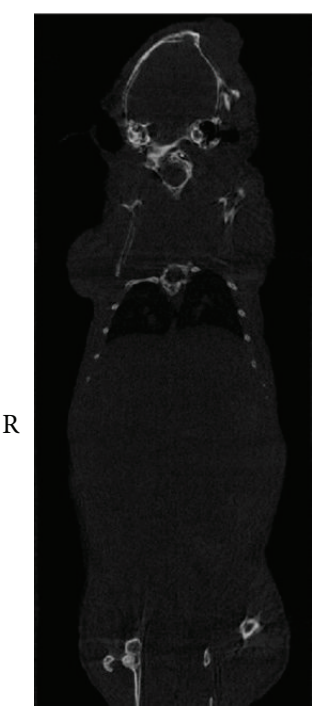

(f)

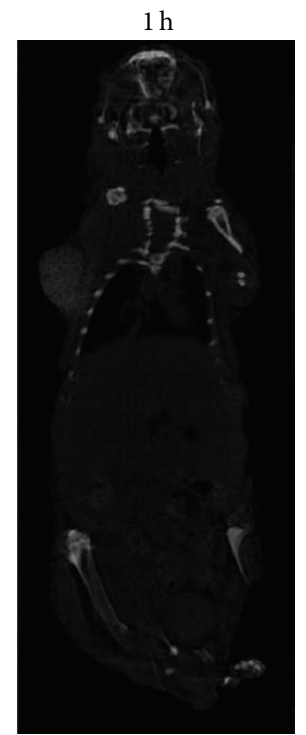

(c)

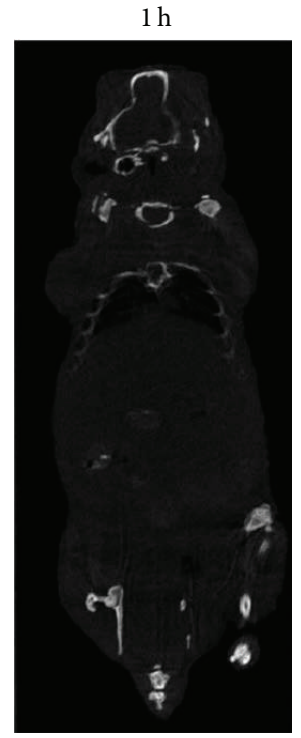

(g)

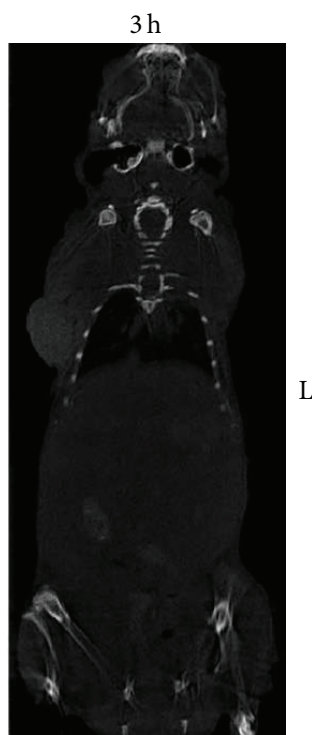

(d)

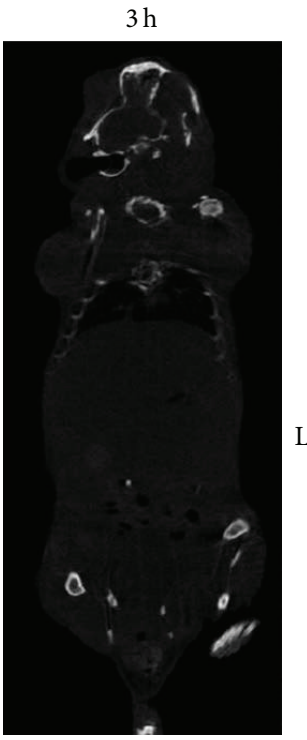

(h)

FIGURE 9: In vivo tumor targeting CT images of mice after intravenous injection with RGD-AuNRs at different time points. (a-d) The CT images of positive group. (e-h) The CT images of negative group. (a, e) The photographs of mice. (b, f) The CT images at $0 \mathrm{~h}$. (c, g) The CT images at $1 \mathrm{~h} .(\mathrm{d}, \mathrm{h})$ The CT images at $3 \mathrm{~h}$.

2004, Hainfeld et al. [32] investigated the high atomic weight of gold in the field of X-ray imaging; most of the examination on inorganic nanoparticles-based X-ray contrasting agents is focused on gold nanoparticles or their hybrids. With lower $\mathrm{X}$-ray dosage, gold has a better X-ray attenuation than iodine. Besides the characteristic that gold nanomaterials display a strong X-ray attenuation, researches have also focused on their use for X-ray CT and multimodal imaging in terms of the substantial control of their physical, chemical, and biological properties [33]. As emphasized above, the applications of gold nanomaterials for CT imaging and cancer diagnosis are superior with the properties of easy self-assembly with specific functional molecules or targeting agents for targeting tumor cells (e.g., antibody, aptamers, and peptides), prolonged circulation time, enhanced renal clearance, noticeable optical properties that could be varied depending on their particle size and shape, and the satisfactory biocompatibility $[20,27,29]$. With the change of the shape, gold nanorods (AuNRs) perform tunable optical properties which could be applied on biomedical targeting, photothermal therapy, and in vivo imaging [30, 31]. More recently, AuNRs as a potential $\mathrm{X}$-ray contrast agent have been used in CT imaging [34]. In the literatures, it has been reported that PEGylated gold nanorods can lead to a prolonged half-life time of $19 \mathrm{~h}$ [35]. 
Kim et al. [36] and Cai et al. [37] exploited in vivo applications of AuNPs as X-ray contrast agents with micro-CT. They managed PEGylted AuNPs as blood pool contrasting agents to provide apparent vascular contrast enhancement effect with plasma half-time around $12 \mathrm{~h}$ and $14.6 \mathrm{~h}$, respectively. On the other hand, to enhance the targeting ability, AuNRs can be functionalized by chemical modification such as DNA, peptides, and antibodies.

In this study, we have prepared a new RGD-AuNRs probe for integrin $\alpha_{v} \beta_{3}$ targeting and tumor imaging. By PEG modification and small molecule peptides RGD conjugation, unspecific binding ability of AuNRs could be dramatically decreased while targeting ability was increased obviously which can benefit the accumulation of AuNRs in tumor tissues and prolonged the residence time in the biological tissues. Also the MTT assay and stability measurement showed that RGD conjugation eliminated their cytotoxicity and improved their biocompatibility and stability. RGD conjugation increased the in vitro integrin $\alpha_{v} \beta_{3}$ binding affinity by the contrast between RGD-AuNRs and PEG-AuNRs. The feasibility of RGD-AuNRs for the noninvasive detection of integrin $\alpha_{v} \beta_{3}$ expression was assessed in both U87 and HT-29 tumor models. And the tumor uptake of AuNRs-RGD was also significantly higher in integrin $\alpha_{v} \beta_{3}$-positive U87 tumor model than in integrin $\alpha_{v} \beta_{3}$-negative HT-29 tumor model.

Since AuNRs stabilized with CTAB show strong cytotoxicity, the management that barely depending on centrifugation will lead to aggregation of AuNRs. Several methods to eliminate the toxicity of AuNRs and stabilize them in physiological environment have been researched. Jain et al. [38] confirmed photothermal therapy and NIR cell imaging by anti-EGFR antibody-conjugated AuNRs which could bound to malignant cancer cells expressing EGFR as light scattering and photothermal therapy agents in vitro. There exist several other methods that have been reported to displace CTAB to modify the surface chemistry and decrease the cytotoxicity of AuNRs, including polyelectrolyte wrapping [39], substitution by a thiol-terminal PEG [25], and alternative by alkanethiols [40] and lipids [41]. Niidome et al. [35] demonstrated the feasibility to reduce the toxicity by PEG modification. In their study, they testified the cell viability of two kinds of AuNRs (stabilized by CTAB and modified with PEG). The results also verified the fact that PEG modification is an effective strategy for preparing biocompatible materials. Here, we managed to replace CTAB with PEG for biological applications. PEG modification was achieved by adding PEG in the AuNRs solution; then, excess PEG was removed by centrifugation. PEG-modified gold nanoparticles showed a nearly neutral surface and had little cytotoxicity in vitro. In our studies, the results suggested that the substitution of CTAB by polyethylene glycol eliminated the cytotoxicity of AuNRs to a large extent (Figure 4 ). It is well known that integrin $\alpha_{v} \beta_{3}$ plays a decisive role in tumor growth, invasion, and metastasis. Gladson [42] and Bello et al. [43] proved the fact that integrin $\alpha_{v} \beta_{3}$ expression occurs in glioma endothelial cells using relatively small numbers of tumor frozen sections. Integrin $\alpha_{v} \beta_{3}$ has a negligible expression on epithelial cells and mature endothelial cells, but it is highly upregulated on the activated endothelial cells of tumor neovasculature and some tumor cells such as human glioblastoma cell line [44, 45]. It is noteworthy that peptides (RGD) could target integrin $\alpha_{v} \beta_{3}$, which can specifically track several kinds of tumors, whereas antibody can only specifically recognize one certain kind of tumor. Haubner et al. [46] studied for the first time the corresponding relationship between the tumor uptake ability of RGD probe and the integrin $\alpha_{v} \beta_{3}$ expression level. And U87 cells were chosen as the integrin $\alpha_{v} \beta_{3}$-positive cells and HT-29 cells were chosen as the integrin $\alpha_{v} \beta_{3}$-negative cells.

In vitro integrin $\alpha_{v} \beta_{3}$ receptor binding ability of RGDAuNRs was analyzed by dark-field imaging experiments. The uptake efficiency of AuNRs-RGD by U87 cells was much higher than HT-29 cells. For U87 cells (positive group) that had a higher expression of integrin $\alpha_{v} \beta_{3}$ than HT-29 cells (negative group), the specific binding of RGD-AuNRs with U87 cells was more obvious (Figure 5). The RGD-AuNRs were tail vein injected into two nude mice tumor models of U87 (integrin $\alpha_{v} \beta_{3}$-positive tumor) and HT-29 (integrin $\alpha_{v} \beta_{3}$-negative tumor) for CT imaging. RGD-AuNRs were capable of specifically recognizing tumor cells and tumor neovasculature with a high expression of integrin $\alpha_{v} \beta_{3}$. The accumulation of RGD-AuNRs at the U87 tumor site was more significant than the HT-29 tumor site. The CT images of the tumor site with a higher contrast than other soft tissues due to the high X-ray attenuation of RGD-AuNRs specifically in the U87 tumor model (Figure 9). Because of the systemic circulation and metabolism in vivo and the character of nanomaterials themselves, some AuNRs intravenously injected could not finally arrive at the tumor site. So compared to the CT imaging of subcutaneous and intramuscular injection of RGDAuNRs into the mouse (Figures 7 and 8), the results were less obvious. Some methods may be effective in making the imaging more clear and increasing the contrast effects. Adding large gold doses is an optional method [47]; thus, amounts suitable for accurate quantification can be used to provide noise-free images clearly. Another factor is the micro-CT unit. By tweaking these preferences, favorable contrast from gold can also be produced owing to its significantly greater attenuation coefficient than soft tissue [47]. Dou et al. demonstrated that the optimal sizes of spherical AuNPs for simultaneous CT imaging and radiosensitization could be selected and evaluated based on the distinctive size-dependent enhancement effects [48]. Regulating the aspect ratio and the size of the RGD-AuNRs that we synthesized may achieve the same goal. The contrast effects of subcutaneous and intramuscular injection of RGD-AuNRs into the mouse were bright enough to verify the feasibility of gold nanomaterials in vivo imaging. This may due to the resistance in other organs during the blood circulation. Niidome et al. [35] verified the biodistribution of surface modified AuNRs after intravenous injection by ICP mass spectrometry at different time points. AuNRs may accumulate in the liver and other major organs like kidney with the flow of blood. Yang et al. [49] investigated the interaction mechanism of nanomaterials with multiple biological tissues. By nanomaterials passivation, hydrophobicity/hydrophilicity, dissolution, and coating, particles may tend to interact with cell membranes and surrounding medium. Thus, due to the systemic circulation and metabolism in vivo, not all intravenously injected AuNRs could 
finally arrive at the tumor site. This explained the phenomenon that the CT imaging of intravenous injection was not satisfied as subcutaneous and intramuscular injection of RGD-AuNRs. In subsequent study, we will focus on the research of the relationship between the best contrast agent loading or concentration and the multimodal imaging of gold nanomaterials.

\section{Conclusion}

The result is noteworthy for biomedicine applications of gold nanomaterials. And most researches have illustrated that the well-established application between strong X-ray attenuation, facile chemical and surface properties, and low toxicity has focused on gold nanoparticles-based systems. But for better clinical use, the targeting ability and multimodal imaging capability need to be further improved. Herein, first of all, compared with the existing iodine containing molecules contrast agents, gold nanorods (AuNRs) that we synthesized are more promising as CT contrast agents for its nontoxic, high contrast, long imaging time, and other advantages. In the second part, the modification of RGD peptide could improve biocompatibility and stability of AuNRs. RGDAuNRs targeting tumor CT imaging has improved the resolution of tumor tissue. RGD-AuNRs could be excreted without toxic side effects. And the RGD-AuNRs based targeting therapy agents can be applied on various tumors with high expression of integrin $\alpha_{v} \beta_{3}$.

In a word, the RGD-AuNRs that we compounded as a significant potential candidate in biomedical applications possess many advantages. First, AuNRs have an adjustable optical property. The longitudinal plasma resonance absorption peaks of RGD-AuNRs that we synthesized was in the near-infrared light region. Second, the strong penetration of near-infrared light in tissues makes it possible to improve the imaging depth of AuNRs. Furthermore, the good biocompatibility and nontoxicity of RGD-AuNRs that we prepared have the capacity to improve the micro-CT imaging and the ability to target U87 glioblastoma tumor models. Due to these properties, their imaging capability has been greatly improved, including CT imaging and optical imaging. Therefore, RGDAuNRs nanoprobes can be excellent materials in biomedical applications such as tumor targeting and imaging.

\section{Competing Interests}

The authors disclose no potential competing interests.

\section{Acknowledgments}

Authors are highly thankful for Professor Shouping Zhu, Jinwang Li, and Lei Xiong in Xidian University and Professor Jing Wang and Shu Zong in Xijing Hospital for their assistance during the CT data acquisition. This work is supported by the Program of the National Basic Research and Development Program of China (973) under Grant no. 2011CB707702, the National Natural Science Foundation of China under Grant nos. 81371589, 81090272, 81101084, 81227901, and
81530058, the Natural Science Basic Research Plan in Shaanxi Province of China under Grant no. 2015JZ019, and the Fundamental Research Funds for the Central Universities (NSIZ021402).

\section{References}

[1] P. Ray, "Multimodality molecular imaging of disease progression in living subjects," Journal of Biosciences, vol. 36, no. 3, pp. 499-504, 2011.

[2] P. Huang, L. Bao, C. Zhang et al., "Folic acid-conjugated silicamodified gold nanorods for X-ray/CT imaging-guided dualmode radiation and photo-thermal therapy," Biomaterials, vol. 32, no. 36, pp. 9796-9809, 2011.

[3] R. Popovtzer, A. Agrawal, N. A. Kotov et al., "Targeted gold nanoparticles enable molecular CT imaging of cancer," Nano Letters, vol. 8, no. 12, pp. 4593-4596, 2008.

[4] L. Li, L. Zhang, T. Wang et al., "Facile and scalable synthesis of novel spherical Au nanocluster assemblies@polyacrylic acid/calcium phosphate nanoparticles for dual-modal imagingguided cancer chemotherapy," Small, vol. 11, no. 26, pp. 31623173, 2015.

[5] X. Qu, Y. Li, L. Li, Y. Wang, J. Liang, and J. Liang, "Fluorescent gold nanoclusters: synthesis and recent biological application," Journal of Nanomaterials, vol. 2015, Article ID 784097, 23 pages, 2015.

[6] A. Antonelli, C. Sfara, E. Manuali, I. J. Bruce, and M. Magnani, "Encapsulation of superparamagnetic nanoparticles into red blood cells as new carriers of MRI contrast agents," Nanomedicine, vol. 6, no. 2, pp. 211-223, 2011.

[7] X. Cui, S. Belo, D. Krüger et al., "Aluminium hydroxide stabilised $\mathrm{MnFe}_{2} \mathrm{O}_{4}$ and $\mathrm{Fe}_{3} \mathrm{O}_{4}$ nanoparticles as dual-modality contrasts agent for MRI and PET imaging," Biomaterials, vol. 35, no. 22, pp. 5840-5846, 2014.

[8] K. Morishige, D. F. Kacher, P. Libby et al., "High-resolution magnetic resonance imaging enhanced with superparamagnetic nanoparticles measures macrophage burden in atherosclerosis," Circulation, vol. 122, no. 17, pp. 1707-1715, 2010.

[9] C. R. Anderson, X. Hu, H. Zhang et al., "Ultrasound molecular imaging of tumor angiogenesis with an integrin targeted microbubble contrast agent," Investigative Radiology, vol. 46, no. 4, pp. 215-224, 2011.

[10] J. K. Willmann, R. H. Kimura, N. Deshpande, A. M. Lutz, J. R. Cochran, and S. S. Gambhir, "Targeted contrast-enhanced ultrasound imaging of tumor angiogenesis with contrast microbubbles conjugated to integrin-binding knottin peptides," Journal of Nuclear Medicine, vol. 51, no. 3, pp. 433-440, 2010.

[11] S. M. Janib, A. S. Moses, and J. A. MacKay, "Imaging and drug delivery using theranostic nanoparticles," Advanced Drug Delivery Reviews, vol. 62, no. 11, pp. 1052-1063, 2010.

[12] T. Reuveni, M. Motiei, Z. Romman, A. Popovtzer, and R. Popovtzer, "Targeted gold nanoparticles enable molecular CT imaging of cancer: an in vivo study," International Journal of Nanomedicine, vol. 6, pp. 2859-2864, 2011.

[13] N. Lee, S. H. Choi, and T. Hyeon, "Nano-sized CT contrast agents," Advanced Materials, vol. 25, no. 19, pp. 2641-2660, 2013.

[14] T. Luo, P. Huang, G. Gao et al., "Mesoporous silica-coated gold nanorods with embedded indocyanine green for dual mode Xray CT and NIR fluorescence imaging," Optics Express, vol. 19, no. 18, pp. 17030-17039, 2011. 
[15] J. Zhang, C. Li, X. Zhang et al., "In vivo tumor-targeted dual-modal fluorescence/CT imaging using a nanoprobe coloaded with an aggregation-induced emission dye and gold nanoparticles," Biomaterials, vol. 42, pp. 103-111, 2015.

[16] A. Jakhmola, N. Anton, and T. F. Vandamme, "Inorganic nanoparticles based contrast agents for X-ray computed tomography," Advanced Healthcare Materials, vol. 1, no. 4, pp. 413-431, 2012.

[17] C. Haller and I. Hizoh, "The cytotoxicity of iodinated radiocontrast agents on renal cells in vitro," Investigative Radiology, vol. 39, no. 3, pp. 149-154, 2004.

[18] I. Hizoh and C. Haller, "Radiocontrast-induced renal tubular cell apoptosis: hypertonic versus oxidative stress," Investigative Radiology, vol. 37, no. 8, pp. 428-434, 2002.

[19] M. Shilo, T. Reuveni, M. Motiei, and R. Popovtzer, "Nanoparticles as computed tomography contrast agents: current status and future perspectives," Nanomedicine, vol. 7, no. 2, pp. 257269, 2012.

[20] http://physics.nist.gov/PhysRefData/XrayMassCoef.

[21] A. Ding, Y. Chen, C. C. Wang, P. Li, and D. Shieh, "HER-2 antibody conjugated gold nano rod for in vivo photothermal therapy," in Proceedings of the 8th IEEE Conference on Nanotechnology (NANO '08), pp. 882-885, IEEE, 2008.

[22] C. J. Murphy, L. B. Thompson, A. M. Alkilany et al., "The many faces of gold nanorods," The Journal of Physical Chemistry Letters, vol. 1, no. 19, pp. 2867-2875, 2010.

[23] P. Yan, N. Zhao, H. Hu, X. Lin, F. Liu, and F.-J. Xu, "A facile strategy to functionalize gold nanorods with polycation brushes for biomedical applications," Acta Biomaterialia, vol. 10, no. 8, pp. 3786-3794, 2014.

[24] D. P. K. Lankveld, R. G. Rayavarapu, P. Krystek et al., "Blood clearance and tissue distribution of PEGylated and nonPEGylated gold nanorods after intravenous administration in rats," Nanomedicine, vol. 6, no. 2, pp. 339-349, 2011.

[25] H. Liao and J. H. Hafner, "Gold nanorod bioconjugates," Chemistry of Materials, vol. 17, no. 18, pp. 4636-4641, 2005.

[26] S. Liu, "Radiolabeled cyclic RGD peptides as integrin $\alpha_{v} \beta_{3}$ targeted radiotracers: maximizing binding affinity via bivalency, Bioconjugate Chemistry, vol. 20, no. 12, pp. 2199-2213, 2009.

[27] Z. Liu, B. Jia, J. Shi et al., "Tumor uptake of the RGD dimeric probe $\mathrm{Tc}^{99 \mathrm{~m}}-\mathrm{G}_{3}-2 \mathrm{P}_{4}-\mathrm{RGD} 2$ is correlated with integrin $\alpha_{v} \beta_{3}$ expressed on both tumor cells and neovasculature," Bioconjugate Chemistry, vol. 21, no. 3, pp. 548-555, 2010.

[28] Z. Liu, J. Shi, B. Jia et al., "Two 90Y-labeled multimeric RGD peptides RGD4 and 3PRGD2 for integrin targeted radionuclide therapy," Molecular Pharmaceutics, vol. 8, no. 2, pp. 591-599, 2011.

[29] B. Nikoobakht and M. A. El-Sayed, "Preparation and growth mechanism of gold nanorods (NRs) using seed-mediated growth method," Chemistry of Materials, vol.15, no. 10, pp. 19571962, 2003.

[30] J. Choi, J. Yang, J. Park et al., "Specific near-IR absorption imaging of glioblastomas using integrin-targeting gold nanorods," Advanced Functional Materials, vol. 21, no. 6, pp. 1082-1088, 2011.

[31] A. J. Gormley, A. Malugin, A. Ray, R. Robinson, and H. Ghandehari, "Biological evaluation of RGDfK-gold nanorod conjugates for prostate cancer treatment," Journal of Drug Targeting, vol. 19, no. 10, pp. 915-924, 2011.
[32] J. F. Hainfeld, D. N. Slatkin, and H. M. Smilowitz, "The use of gold nanoparticles to enhance radiotherapy in mice," Physics in Medicine and Biology, vol. 49, no. 18, pp. N309-N315, 2004.

[33] H.-X. Xia, X.-Q. Yang, J.-T. Song et al., "Folic acid-conjugated silica-coated gold nanorods and quantum dots for dualmodality CT and fluorescence imaging and photothermal therapy," Journal of Materials Chemistry B, vol. 2, no. 14, pp. 1945-1953, 2014.

[34] J. F. Hainfeld, D. N. Slatkin, T. M. Focella, and H. M. Smilowitz, "Gold nanoparticles: a new X-ray contrast agent," The British Journal of Radiology, vol. 79, no. 939, pp. 248-253, 2006.

[35] T. Niidome, M. Yamagata, Y. Okamoto et al., "PEG-modified gold nanorods with a stealth character for in vivo applications," Journal of Controlled Release, vol. 114, no. 3, pp. 343-347, 2006.

[36] D. Kim, S. Park, J. H. Lee, Y. Y. Jeong, and S. Jon, "Antibiofouling polymer-coated gold nanoparticles as a contrast agent for in vivo X-ray computed tomography imaging," Journal of the American Chemical Society, vol. 129, no. 24, pp. 7661-7665, 2007.

[37] Q.-Y. Cai, S. H. Kim, K. S. Choi et al., "Colloidal gold nanoparticles as a blood-pool contrast agent for X-ray computed tomography in mice," Investigative Radiology, vol. 42, no. 12, pp. 797-806, 2007.

[38] P. K. Jain, K. S. Lee, I. H. El-Sayed, and M. A. El-Sayed, "Calculated absorption and scattering properties of gold nanoparticles of different size, shape, and composition: applications in biological imaging and biomedicine," The Journal of Physical Chemistry B, vol. 110, no. 14, pp. 7238-7248, 2006.

[39] A. Gole and C. J. Murphy, "Polyelectrolyte-coated gold nanorods: synthesis, characterization and immobilization," Chemistry of Materials, vol. 17, no. 6, pp. 1325-1330, 2005.

[40] Z. Sun, W. Ni, Z. Yang, X. Kou, L. Li, and J. Wang, "pH-controlled reversible assembly and disassembly of gold nanorods," Small, vol. 4, no. 9, pp. 1287-1292, 2008.

[41] Y. Niidome, K. Honda, K. Higashimoto et al., "Surface modification of gold nanorods with synthetic cationic lipids," Chemical Communications, no. 36, pp. 3777-3779, 2007.

[42] C. L. Gladson, "Expression of integrin $\alpha \nu \beta 3$ in small blood vessels of glioblastoma tumors," Journal of Neuropathology \& Experimental Neurology, vol. 55, no. 11, pp. 1143-1149, 1996.

[43] L. Bello, M. Francolini, P. Marthyn et al., " $\alpha \mathrm{v} \beta 3$ and $\alpha \mathrm{v} \beta 5$ integrin expression in glioma periphery," Neurosurgery, vol. 49, no. 2, pp. 380-390, 2001.

[44] M. Bartneck, H. A. Keul, M. Wambach et al., "Effects of nanoparticle surface-coupled peptides, functional endgroups, and charge on intracellular distribution and functionality of human primary reticuloendothelial cells," Nanomedicine: Nanotechnology, Biology, and Medicine, vol. 8, no. 8, pp. 1282-1292, 2012.

[45] Z. Li, P. Huang, X. Zhang et al., "RGD-conjugated dendrimermodified gold nanorods for in vivo tumor targeting and photothermal therapy," Molecular Pharmaceutics, vol. 7, no. 1, pp. 94-104, 2010.

[46] R. Haubner, W. A. Weber, A. J. Beer et al., "Noninvasive visualization of the activated $\alpha \mathrm{v} \beta 3$ integrin in cancer patients by positron emission tomography and $\left[{ }^{18} \mathrm{~F}\right]$ Galacto-RGD," PLoS Medicine, vol. 2, no. 3, article e70, 2005.

[47] J. F. Hainfeld, M. J. O'Connor, F. A. Dilmanian, D. N. Slatkin, D. J. Adams, and H. M. Smilowitz, "Micro-CT enables microlocalisation and quantification of Her2-targeted gold nanoparticles within tumour regions," British Journal of Radiology, vol. 84, no. 1002, pp. 526-533, 2011. 
[48] Y. Dou, Y. Guo, X. Li et al., "Size-tuning ionization to optimize gold nanoparticles for simultaneous enhanced CT imaging and radiotherapy," ACS Nano, vol. 10, no. 2, pp. 2536-2548, 2016.

[49] R. S. H. Yang, L. W. Chang, J.-P. Wu et al., "Persistent tissue kinetics and redistribution of nanoparticles, quantum dot 705, in mice: ICP-MS quantitative assessment," Environmental Health Perspectives, vol. 115, no. 9, pp. 1339-1343, 2007. 

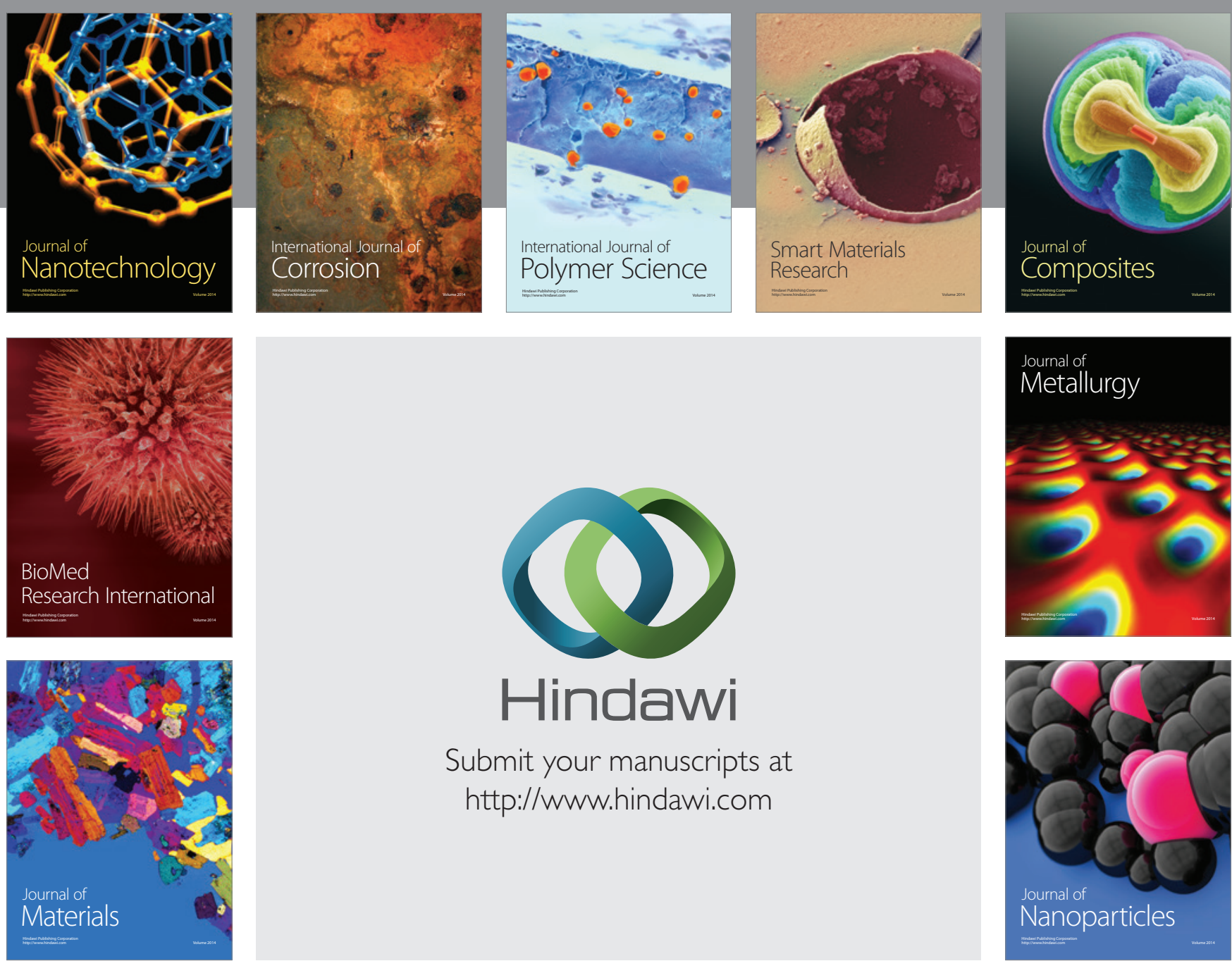

\section{Hindawi}

Submit your manuscripts at

http://www.hindawi.com

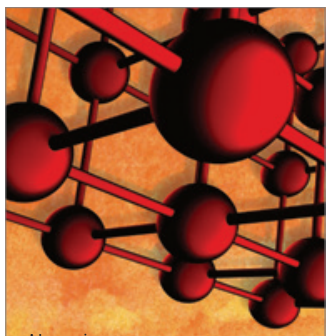

Materials Science and Engineering
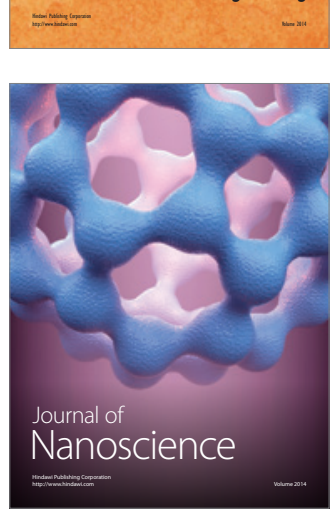
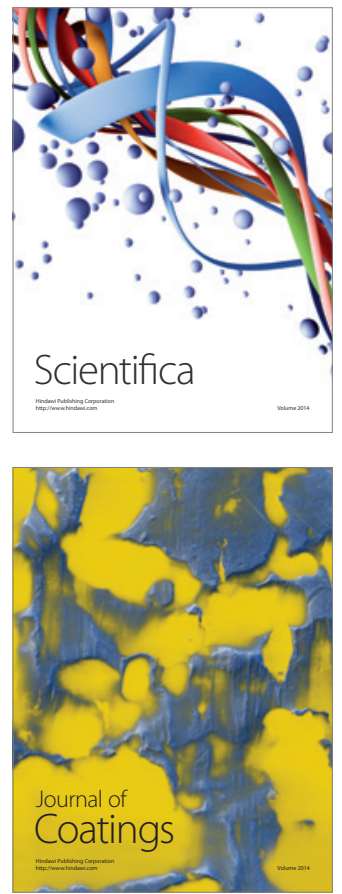
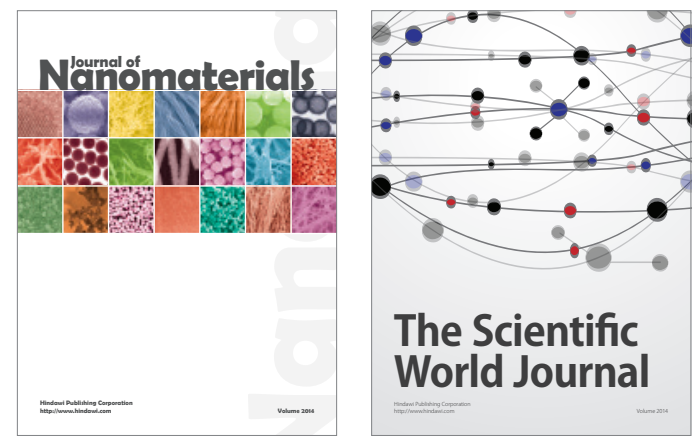

The Scientific World Journal
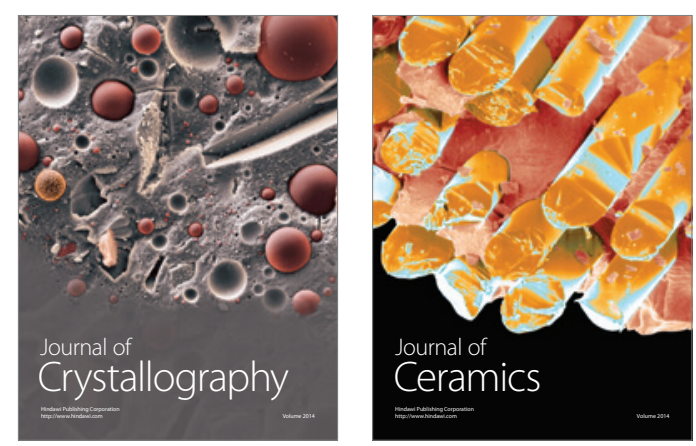
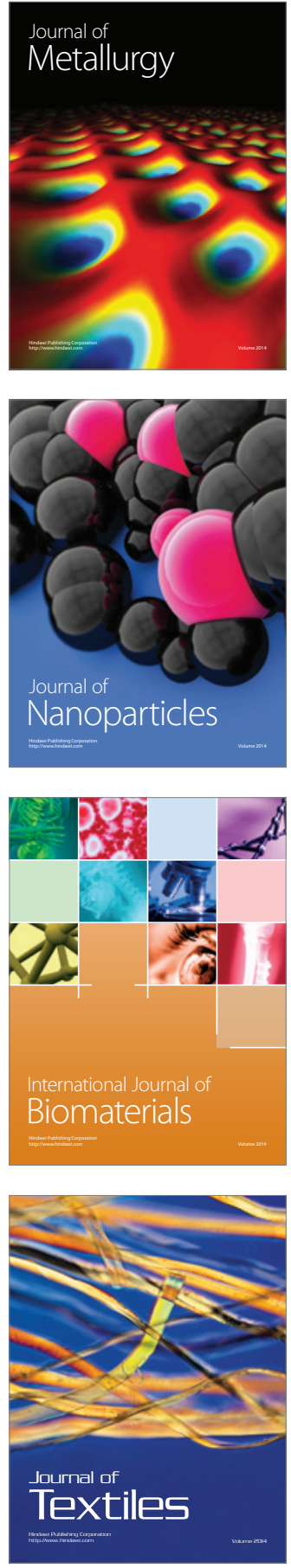